\title{
Translated Abstracts
}

To advance dissemination of psychotherapy integration scholarship internationally, Chinese and Spanish translations of the title, abstract, and keywords associated with articles appearing in the Journal of Psychotherapy Integration are provided. Articles are listed below in order of their appearance in this issue.

\section{Redefining the Future of SEPI: Member Characteristics, Integrative Practices, and Organizational Satisfactions \\ John C. Norcross, Bryan M. Nolan, Dana C. Kosman, and Hector Fernández-Alvarez. http://dx.doi.org/10.1037/int0000051}

Redefiniendo el futuro de SEPI: Característicos de los miembros, prácticas integradas, y satisfacciones organizacionales

Todos los miembros de la Sociedad para la Exploración de la Integración Psicoterapéutica (SEPI, por sus siglas en inglés) recibieron una encuesta electrónica $(N=335 ; 55 \%$ tasa de respuesta) en enero de 2016, preguntando sobre sus características sociodemográficas, sus compromisos teóricos, sus prácticas integradas, sus satisfacciones organizacionales y recomendaciones para direcciones futuras. Éste artículo resume los resultados de dicha encuesta y provee un cuadro contemporáneo de los miembros de SEPI, y cuando posible, una comparación histórica con los resultados de un estudio similar llevado a cabo en el 1996. La imagen emergente es de una asociación que ésta en crecimiento demográfico e de diversidad internacional (53\% mujeres, 39\% miembros fuera de E.U.A.) entre profesionales de salud mental trabajando en puestos universitarios y prácticas independientes. Las teorías psicodinámicas, humanísticas, y cognitiva-comportamental fueron las más que influenciaron las prácticas integradas de los encuestados. Los subtipos de integración más frecuentes fueron esos de integración asimilativa, integración teórica, y factores comunes. Los miembros estaban contundentemente satisfechos con la organización en general, la revista, y las conferencias anuales; comparado con el 1996 los encuestados estaban marcadamente más satisfechos con los recursos de la asociación en el 2016. Sin embargo, miembros mostraron menos entusiasmo en respecto a las redes regionales. A la vez, 8 recursos del SEPI eran desconocidos para la mayoría de los miembros. Miembros reportaron satisfacción con el progreso en la mayoría de las prioridades. Discutimos en éste artículo las implicaciones de estos resultados y recomendaciones para mejorías.

Claves: integración psicoterapéutica, Sociedad para la Exploración de la Integración Psicoterapéutica, integración, factores comunes, orientaciones teóricas

\section{重新定义SEPI的未来：成员特征, 整合化实践和组织满意度}

我们在2016年一月对“心理治疗一体化探索学会” (SEPI)。所有会员进行了其社会人口特征, 理论承诺, 整合化实践, 组织满意度, 以及对未来方向的建议的电子 (问卷) 调查 (样本大小 $=335$ 名, $55 \%$ 回应率/ 反应率) 本文总结了这些 (调查) 结果, 并提供了对于SEPI会员的当代图景, 以及, 在可能 地情况下, 与1996年一个相似的研究结果进行了历史对比。得到的图景是一个越来越在社会人口方 面和国际性方面多样化的, 受聘于高校以及独立诊所的心理健康专业人士们的学会 $(53 \%$ 女性, $39 \%$ 非美籍会员)。精神动力学, 人本主义, 以及认知行为理论对应答者的整合化实践影响最大。最 常见的 / 频繁的整合化子类型是同化性整合化, 理论性整合化, 以及共同因素。会员们对于(学会的) 整体结构, 其期刊, 以及年会空前地 / 压倒性地满意; 2016年应答者对于学会资源的满意度要远 远高于1996年。但是, 会员们对于区域性网络则没有那么热情。同时, 大部分会员对八个SEPI资源并 不知情。会员们对大部分优先事宜的进展表示满意。(最后我们) 讨论了这些结果的影响以及改进 建议。

\section{关键词 心理治疗一体化 探索心理治疗一体化学会 共同因素 理论导向}

The Proportion of Integrationists Among Czech Psychotherapists and Counselors: A Comparison of Multiple Criteria

Tomas Rihacek and Jan Roubal

http://dx.doi.org/10.1037/int0000069

La proporción de integrativos entre psicoterapeutas y consejeros checos: Una comparación de criterios multiples 
La integración o el eclecticismo de un psicoterapeuta puede operar en diferentes maneras. Estudios típicamente han estimado la proporción de integrativos/eclécticos utilizando un solo criterio, frecuentemente rindiendo resultados incomparables. El objetivo de éste estudio fue estimar la frecuencia de integración/ eclecticismo utilizando 4 criterios distintos basados en entrenamiento psicoterapéutico, orientación teórica auto-identificada, y el uso de técnicas psicoterapéuticas. El análisis se basó en data obtenida a través de un cuestionario en-línea completado por 373 psicoterapeutas o consejeros checos. Estimamos la proporción de practicantes integrativos a $21.7 \%, 32.7 \%, 87.7 \%$ y $98.9 \%$ dependiendo del criterio utilizado. Adicionalmente, estimamos el endoso de diferentes tipos de integración y exploramos la frecuencia de combinaciones de las orientación teóricas primarias. Discutimos los resultados colocándolos en el contexto de estudios previos y damos énfasis al rol de la operacionalización adecuada de la integración. Los resultados sugieren que a pesar de que la orientación teórica auto-identificada puede reflejar la identidad profesional del practicante, medir el nivel de técnicas puede ser más sensible a la integración real de varias orientaciones.

Claves: Eclecticismo, operacionalización, técnicas, integración psicoterapéutica, orientación teórica

捷克心理治疗师和心理咨询师中整合主义者的比例: 多重标准的比较

一个心理治疗师的整合 / 折衰主义能够以不同的方法来操作 (定义)。现有估计整合主义者/折衰主 义者比例的研究通常只使用一种标准, 而这常常导致不可比较性结果。本研究的目标是使用不同的四 种基于心理治疗培训, 自认的理论倾向, 以及对心理治疗技术应用 / 使用的标准来估计整合主义 / 折衰主义的盛行率 / 流行率。本分析基于通过在线调查从 373 捷克心理治疗者和心理咨询师获 取的问卷数据。根据使用的标准 (的不同), 整合者的比例估计为 $21.7 \%, 32.7 \%, 87.7 \%$ 以及 $98.9 \%$ 。 此外 / 而且, 本研究估计了对不同类型的整合化的认可 / 支持的比例, 并探索了主要理论倾向 组合的频率。我们在以往研究的背景下讨论了我们的研究结果, 并强调了充分的操作化整合主义的 的作用 / 角色。结果显示/暗示尽管自评价的理论倾向可以反映从业者的专业身份和 (其) 隶属 / 立场, 技术水平的测量也许 / 可能更敏感地反映不同倾向在现实中的整合化。

关键词 折衰主义 操作化 技术 心理治疗一体化 理论导向

Client Perceptions of Corrective Experiences in Cognitive Behavioral Therapy and Motivational Interviewing for Generalized Anxiety Disorder: An Exploratory Pilot Study

Jasmine Khattra, Lynne Angus, Henry Westra, Christianne Macaulay, Kathrin Moertl, and Michael Constantino

http://dx.doi.org/10.1037/int0000053

Percepciones de clientes sobre experiencias correctivas en la terapia cognitiva-conductual y la entrevista motivacional para el trastorno de ansiedad generalizada: Un estudio piloto exploratorio

El objetivo del estudio presente fue explorar cualitativamente los reportes pos-terapia de clientes luego de experiencias correctivas - un factor común propuesto y principio integrativo de la terapia de cambio (Castonguay \& Hill, 2012) - luego de completar terapia cognitiva-conductual breve (CBT, por sus siglas en inglés) o entrevista motivacional (MI, por sus siglas en inglés) integrada con CBT (MI-CBT) para trastornos de ansiedad generalizada (GAD, por sus siglas en inglés; Westra, Constantino, \& Antony, 2016). Entrevistas semi-estructuradas fueron llevadas a cabo (Percepciones de pacientes sobre experiencias correctivas en terapia individual, PPCEIT; Constantino, Angus, Friedlander, Messer, \& Heatherington, 2011). Estas fueron completadas luego de la terminar la terapia con 1 clienta MI-CBT y 1 clienta recibiendo solo CBT, ambos que cumplían con el criterio para recuperación. Las entrevistas PPCEIT fueron grabadas en audio, transcritas y sometidas para un análisis de teoría fundamentada utilizando un programa software de método de investigación cualitativa (ATLAS.ti). Resultados indicaron que ambas clientas reportaron cambios positivos en su experiencia de ansiedad y mayor acción en sus relaciones interpersonales. Específicamente, la clienta completando tratamiento integrativo MI-CBT reportó mayor confianza en su habilidad para mantener cambios positivos pos-terapia, mientras que la clienta que recibió solo CBT expresó confianza en su aplicación de herramientas CBT y destrezas para mantener los resultados de la terapia. La clienta MI-CBT atribuyó los cambios que ella experimentó en terapia a una conciencia aumentada y mayor confianza en sus propia agencia, indicando una posible experiencia correctiva del ser. Mientras tanto, la clienta recibiendo solamente CBT atribuyó los cambios positivos que ella experimentó al la pericia del terapista. Discutimos direcciones futuras para investigación, en adición a las implicaciones de la integración de enfoques CBT para mejorar resultados clínicos.

Claves: experiencias correctivas, terapia cognitiva-conductual, entrevista motivacional, trastorno de ansiedad generalizada, cambios en terapia 
病人 / 客户 / 来谈者对用于治疗广泛性焦虑症的认知行为治疗和动机性访谈 / 面谈中矫正体验的看法: 一个探索性初步 / 预备研究

本研究的目的是在来谈者完成一个对广泛性焦虑症适用的简短的认知行为治疗 (CBT) 或整合动 机性采访(MI)的认知行为治疗 (MI-CBT) (Westra, Constantino, \& Antony, 2016) 后, 定性调查来访者 / 来 谈者 / 病人治疗后的纠正经验的描述。纠正体验被提议为引起因治疗而产生的改变的共同因素和整 合性原则 (Castonguay \& Hill，2012)。在治疗终结时，符合治愈标准的一名接受MI-CBT治疗 的来访者和一名只接受CBT治疗的来谈者完成了一个叫做“病人对个体治疗中的矫正体验的感知” (PPCEIT; Constantino, Angus, Friedlander, Messer, \& Heatherington, 2011) 的半结构化的面谈。我们录音 并转录了该面谈内容, 并用定性研究方法软件(ATLAS.ti)进行了扎根理论分析。结果显示两名来访 者均报告他们的焦虑体验有了正向转变, 而且人际关系中有了更强的控制感/自信。尤其是, 接受整合 的MI-CBT来谈者表示, 她对于自己保持治疗后积极变化的能力的自信有所增加, 而只接受CBT的来 谈者表示了其对于运用认知行为工具和技巧来保持治疗效果的信心。接受MI-CBT的来谈者将她所 经历的积极变化归功于 (其) 增强的意识和对她自己的信心, 而这表明潜在的自我纠正体验。而只接 受CBT的来谈者则将其体验的积极变化归功于治疗师提供的专门知识。我们讨论了未来的研究方向以 及整合性CBT方案对更好的临床效果 / 结果的含义 / 影响 。

\section{关键词 纠正经验 / 体验 认知行为治疗 动机性访谈 广泛性焦虑症 治疗效果}

Guidelines for Reflective Practice in Psychotherapy: A Reflection on the Benefits of Combining Moment-by-Moment and Phase-by-Phase Mapping in Clinical Decision Making

Joana F. Ferreira, Michael Basseches, and António B. Vasco

http://dx.doi.org/10.1037/int0000047

Guía para la práctica reflexiva en la psicoterapia: Una reflexión sobre las ventajas de combinar el trazado de "momento por momento" y "fase por fase" en el manejo de decisiones clínicas

Éste artículo intenta destacar la importancia de la práctica reflexiva, explorar las ventajas de integrar los principios guías del "momento por momento" y "fase por fase" durante la conceptualización y intervención en nuestros casos, y ofrecer a todo tipo de psicoterapeutas un guía para la práctica reflexiva basada en ésta integración. La práctica reflexiva incluye la observación cercana a las respuestas de los pacientes, y la utilización de esas observaciones para considerar opciones que lleven a mayor sintonización terapéutica y a una terapia más exitosa. La integración del "momento por momento" y "fase por fase", al permitir coordinación entre niveles micros y macros, similar a la idea de figura y motivo, favorece a la respuesta del terapista, el monitoreo de progreso y/o encallamiento clínico en los casos presentes y la toma de decisiones clínicamente integrada. Las guías intentan dirigirse hacia el emparejamiento entre las estrategias objetivas del terapista y las capacidades del paciente, y hacia el observar cada momento, en cada fase terapéutica, si y como el paciente es capaz de utilizar los recursos que el terapista ofrece.

Claves: Práctica reflexiva, proceso terapéutico, monitoreo y secuencia

心理治疗中反思性实践指南 / 准则 / 指导方针: 对于临床决策中整合逐时段和逐阶段计划的益处 的反思

本文意在强调反思性实践的重要性, 探索, 在仔细考虑并介入到我们的案例中时, 整合逐时段的和逐 阶段的指导原则 / 纲领的益处, 并为心理治疗师提供基于这种整合的反思性实践的说服指南。反思 性实践包括对于来访者 / 病人 / 来谈者反应的仔细的观察和这些数据的运用以考虑可能引起更好的协 调和更成功的治疗的选择。通过允许, 类似于图形与背景的概念的, 更微观和更宏观的水平间的协调, 来整合逐时段和逐阶段的计划, 有利于治疗师的响应能力, 跟踪进行中的心理治疗的进展或者困境, 和综合临床决策。本文所提供的指南结合了对治疗师的战略目标与患者不断发展的能力之间的匹 配的涉及, 和对每个治疗阶段中的每一时刻的,对于患者是否能够以及如何利用治疗师提供的资源 的观察。

\section{关键词 反思性实践 治疗过程 跟踪和进展}

Adaptation of the Motive-Oriented Therapeutic Relationship Scale to Group Setting in DialecticalBehaviour Therapy for Borderline Personality Disorder

Sabine Keller, Dominique Page, Yves de Roten, Jean-Nicolas Despland, Franz Caspar, and Ueli Kramer http://dx.doi.org/10.1037/int0000061 
Adaptación de La Relación Terapéutica orientada/centrada sobre los motivos orientada a un contexto de grupo en la terapia dialéctica-conductual para el trastorno límite de la personalidad

La relación terapéutica es estudiada como un proceso frecuentemente en la terapia individual y menos en la terapia de grupo. Una razón para la escasez de este tipo de investigación puede ser la metodología complicada que es necesaria para llevar a cabo estudios sobre el proceso en terapia de grupo. La terapia dialécticaconductual (DBT, por sus siglas en inglés) para el trastorno límite de la personalidad (BPD, por sus siglas en inglés), utiliza el contexto del grupo como técnica terapéutica. El propósito de este estudio es desarrollar una versión grupal de una medida de proceso que ha sido exitosamente utilizada en la terapia individual - la escala de la relación terapéutica de motivación orientada (MOTR, por sus siglas en inglés). La misma está basada en conceptualizaciones individuales de casos utilizando un enfoque de Plan de Ejecución. Para llevar esto acabo, 10 sesiones de terapia grupal de destrezas para DBT fueron analizadas de una base de datos dentro de una prueba controlada aleatorizada. En esto se incluyeron 3 pacientes que completaron terapia y 2 terapistas. Los terapistas desconocían de MOTR. Los resultados mostraron que a la adaptación de MOTR para el grupo de destrezas DBT fue factible. La adaptación mostró diferencias de los terapistas en su utilización de MOTR al comparar los diferentes pacientes: terapistas mostraron niveles mayores de MOTR con un paciente comparado con otro. Resultados generales sugieren que terapistas eficaces en el entrenamiento de destrezas DBT utilizan niveles bajos de MOTR, y gran variabilidad de MOTR durante una sesión. Concluimos que la adaptación del instrumento-MOTR a la terapia de grupo es factible y rinde resultados significativos. Por lo tanto, esta escala se puede utilizar en la investigación de procesos en terapia grupal, en particular cuando se desea tener una medida individual de la relación terapéutica.

Claves: Terapia grupal, relación La Relación Terapéutica orientada/centrada sobre los motivos, terapia dialéctica-conductual, trastorno límite de la personalidad, proceso

\title{
边界型人格障碍的辩证行为治疗中“动机导向的治疗关系量表”对群组环境的适用性
}

治疗关系, 作为流程, 通常在个体治疗中研究, 而不常在群组治疗中研究。研究缺乏的一个原因可 能是在群组治疗的流程研究中所必需的复杂方法论 / 学。运用群组作为治疗 (方案) 的一部分的治疗 方法之一是用于 (治疗) 边界型人格障碍 (BPD) 的辩证行为治疗 (DBT)。本研究的目的是开发一 个群组版的流程量表, “动机导向的治疗关系 (MOTR) 量表”。该量表基于使用计划分析方法的个体 化的个案概念化, 并已经成功地用于个体治疗。为此, 我们在随机对照试验的综合数据中抽出并分 析了10次DBT技能群组治疗。这包括了治疗完成者: 3 名患者和 2 名治疗师。治疗师并不知道 MOTR。结果表明, 适用MOTR到DBT技能群组治疗是可行的。通过比较不同患者, 它的适应性显 示了治疗师在使用MOTR的差异：与其中一个患者相比，治疗师对另一个患者呈现另更高程度 的MOTR。总体结果表明有效的治疗师在DBT技能训练中的干预有着相当低的MOTR平均值 和较大的MOTR治疗中变量性 / 变异性。我们得出结论, 适应MOTR量表到群组治疗是可行的, 并会产生有意义的结果。因此, 此量表可以用于群组治疗的流程研究中, 特别是当 (研究者) 希望得到治疗关系的个体化测量的时。

关键词 群组治疗 动机导向的治疗关系 辩证行为治疗边缘型人格障碍 过程

\author{
Relation and Technique in Psychotherapy: Two Partly Overlapping Categories \\ Lars-Gunnar Lundh \\ http://dx.doi.org/10.1037/int0000068
}

Relación y Técnicas en Psicoterapia: Dos Categorías Parcialmente Superpuestas

Los términos "relación" y "técnica" se usan con frecuencia cuando se discuten las partes que hacen a la psicoterapia efectiva, pero se asume que estos términos se refieren a fenómenos conceptualmente diferentes. Este artículo cuestiona la dicotomía entre los términos "relación” y "técnica” basándose en un análisis teórico de los conceptos y la variedad que existe en los fenómenos a los que se refiere. La palabra técnica se refiere a los procedimientos prescriptos para poder llegar a adquirir una meta. Hay diversos métodos para adquirir una técnica como el entrenamiento, la educación y el aprendizaje, y que resulta en la adquisición de las habilidades y actitudes. Argumentamos que existen dos categorías básicas de técnicas en la psicoterapia: (a) técnicas relacionales que son usadas explícitamente o implícitamente con el paciente por el terapeuta y (b) autotécnicas que el terapeuta le enseña al paciente para uso propio (o se aplica a sí mismo). Adicionalmente, argumentamos que la relación terapéutica implica varios aspectos no-técnicos que tienen que ser evaluados sistémicamente. Este modelo de técnica y relación como categorías parcialmente superpuestas es ilustrado con ejemplos de terapia psicodinámica, cognitiva-conductual y humanista-vivencial. Argumentamos que la 
creación de una teoría de psicoterapia se beneficiaría de una búsqueda sistemática a través de la literatura para identificar las técnicas que fueron propugnadas por varios terapeutas y el uso de análisis temáticos para analizar el contenido de estas técnicas en términos de habilidades y actitudes.

Claves: psicoterapia, relación, el concepto de técnica, destrezas, integración psicoterapéutica

心理治疗的关系和技术 (技巧; 方法): 部分重叠的两个类别

术语“关系”和“技术”经常用于讨论哪一个在心理治疗中有效，但这种讨论常常建立在他们指不同 现象的假设之上。本文章对“关系”和“技术”之间的这种二分法提出了疑问。这种质疑是基于对技术 和关系的概念以及它们所指的多样的现象的理论分析完成的。技术被定义为用来实现某一目 标的手段的步骤 / 程序 / 过程。这些步骤通过各种培训, 教育和学徒训练来提供与个人, 结果导致 技能和态度的获取 / 习得。我们认为，心理治疗中有两种基本的技术类别：(a) 关系技术，治疗师在 与患者的互动中明确或隐含地使用关系技术，和 (b) 自我技术，治疗师教患者使用自我技术 (或应用于(治疗师)他/她自己)。此外, 我们认为治疗关系涉及需要系统解决的一些非技术方面。 心理 动力学疗法, 认知行为疗法和人文经验疗法的例子被用来说明这种技术和关系作为部分重叠的类别 的模式。我们认为, 全面的心理治疗理论的发展将受益于系统的文献搜索以找出各种治疗师所倡导 的技术, 以及专题分析的使用以分析这些技术在技能和态度方面的内容。

关键词 心理治疗 关系 技术的概念技能 心理治疗一体化

Anger and Social Fragmentation: The Evil Violence Tunnel

David Allen, Keva Bethell, and Marie Allen-Carroll

http://dx.doi.org/10.1037/int0000022

Enojo y Fragmentación Social: el Túnel de la Violencia Mala

Las Bahamas, como muchos países en la región, ha sido sometida a serios procesos de fragmentación social a causa de la extensión de la epidemia de la cocaína en los 80s y sus continuas secuelas, así como también la significativa recesión financiera internacional. Manifestada por crecientes tasas de crímenes violentos y asesinatos, la fragmentación social es asociada con la expansión de raptos de enojo y la destrucción de la familia y la comunidad. A través de la intervención del grupo de proceso de la comunidad llamado, La familia: Gente Ayudando a Gente (The Family: People Helping People Project), hemos intentado contrarrestar la fragmentación y mejorar la resocialización. En este proceso de intervención, muchas personas que habían sufrido enojo violento compartieron un fenómeno que llamamos el Túnel de la Violencia Mala. Este fenómeno está asociado con la auto-destrucción (suicidio) así como también causa de muerte de otros (homicidio). Este artículo discute (a) la dinámica del Túnel de la Violencia Mala, (b) precursores y provocaciones del Túnel, (c) etapas del Túnel, (d) viñetas sobre el caso y (e) posibles métodos de prevención.

Claves: enojo, fragmentación social, rabia, vergüenza, cambio de percepción

愤怒和社会分裂: 邪恶暴力隧道

巴哈马与该区域许多国家一样,

由于1980年代广泛的可卡因流行 / 泛滥和持续的后遗症 / 遗患以及重大的国际金融衰退, 已经经历 了严重的社会分化过程。快速增长的的谋杀率和暴力犯罪率显示, 社会分裂与广泛的愤怒的爆 发以及家庭和社区的破坏相关联。通过一个称为“家庭: 人民帮助人民工程 / 计划”的社区团体过 程介入措施, 我们致力于抵消 / 反制 / 抵制社会分裂并加强再社会化 (重新社会化)。在这种介 入措施过程中, 经历过暴怒的许多人都有一种我们称之为“邪恶暴力隧道”的现象。它与强烈的 自我毁灭 (自杀) 以及对他人的伤害 (杀人) 的原因 / 出现有关。本文讨论 (a) 邪恶暴力隧道的动态, (b) 隧道的前兆和触发原因 / 因素, (c) 隧道的阶段, (d) 案例和 (e) 可行的预防方案 / 方法。

关键词 愤怒 社会分裂 情绪激怒 耻辱 感知转移

Integration and Clinical Demonstration of Active Ingredients of Short-Term Psychodynamic Therapy for Depression

Liat Leibovich and Sigal Zilcha-Mano

http://dx.doi.org/10.1037/int0000043

Claves: Integración y Demostración Clínica de los Ingredientes Activos de la Terapia Psicodinámica a Corto Plazo para la Depresión 
La terapia psicodinámica para la depresión ha recibido mucho apoyo científico en los últimos años, y hoy en día puede designarse como efectiva (Leichsenring, Leweke, Klein, \& Steinert, 2015). A pesar de que la teoría psicodinámica y la terapia son extensamente usadas clínicamente, los protocolos empíricos de la terapia psicodinámica siguen sin ser predominantes en el trabajo clínico cotidiano. Hasta los últimos años, no han habido intentos sistemáticos para integrar los elementos centrales de los diferentes manuales dinámicos con teorías dinámicas centrales e investigación. En este artículo, integramos los ingredientes activos de las diferentes teorías psicodinámicas, combinando principios centrales a las teorías como Kohut con los tratamientos de manualizados, tal como Terapia de Soporte Expresivo, Terapia Breve Relacional, Terapia dinámica interpersonal y Psicoterapia Dinámica de Apoyo. Dos conceptos basados en la evidencia fueron usados para integrar varios ingredientes activos del tratamiento psicodinámico: la alianza terapéutica y el método del Tema Central de Conflicto Relacional. Tres casos clínicos demostraron los elementos activos más importantes en esta terapia psicodinámica integrada.

Claves: tratamiento psicodinámico, depresión, alianza, Tema Central de Conflicto Relaciona CCRT

\section{抑有症治愈中短期心理动力学疗法的有效成分的整合和临床演示}

抑有症的心理动力学治疗近年来已经得到了相当多的研究支持, 并且目前可以被指定为有效 (Leichsenring, Leweke, Klein, \& Steinert, 2015)。虽然心理动力学理论和治疗广泛应用于临床实践, 但受研究支 持的心理动力学治疗方案在日常临床工作中仍然不普遍 。直到最近, 没有一个完整的系统化的尝 试将不同动态手册的核心要素与核心动态的理论及研究相结合。在这篇文章中, 我们整合了不 同心理动力疗法的有效成分，结合像Kohu理论的核心原则和，诸如支持表达治疗，简短关系治疗， 动态人际治疗和动态支持心理治疗的, 已手册化的治疗方案。两个 (研究) 证据支持的概念被用 来整合各种心理动力学疗法的有效成分: 治疗联和与核心冲突关系主题 (CCRT) 方法。三个 临床案例研究展示了这种一体化心理动力学疗法的最重要的有效要素。

关键词 心理动力学治疗抑郁症 治疗联盟 核心冲突关系主题

Knowing-in-Relation: How Experienced Therapists Integrate Different Sources of Knowledge in Actual Clinical Practice

Hanne Weie Oddli and John McLeod

http://dx.doi.org/10.1037/int0000045

Saber en Relación: La Manera en la cual Terapeutas con Experiencia Integran Diferentes Fuentes de Sabiduría en su Práctica Clínica.

El presente artículo elabora los conceptos de la integración de la psicoterapia abarcando el problema desde la perspectiva de la actividad del terapeuta en las sesiones de terapia. También se lleva a cabo un análisis de información cualitativa sobre el comportamiento durante la sesión y reflexiones posttratamiento de terapeutas con un alto nivel de experiencia y pluralistamente informados. El concepto de "saber en relación" surgió como método para representar la integración momento a momento de la sabiduría y la práctica que fue observada. Se cree que la integración puede ser considerada una actividad que evoluciona y que es procesual, así como también, una implementación de modelos integradores específicos.

Claves: terapista con experiencia, integración, investigación cualitativa, análisis secundario, proceso terapéutico

关联性认识: 经验丰富的治疗师如何将不同的知识来源整合到实际临床实践中

本文通过从治疗过程中临床医生活动的角度来阐述心理治疗整合化的概念。(我们) 对经验丰富并具 有多元化知识的治疗师在心理治疗中的行为以及治疗后的反思进行了定性数据的二级分析。“关联 性认识 (通过关联的认识) ”的概念作为展现 (我们) 观察到的知识和实际的时刻整合/结合的 手段浮现了出来。我们认为, 整合化可以被认为是一个不断发展的过程性活动, 同时也是一种对 特定整合化模型的使用 / 应用 / 实践。

关键词 经验丰富的/有经验的治疗师 整合 / 结合 定性研究 二级分析 治疗过程 